\title{
Malignant triton tumor
}

INSERM

\section{Source}

INSERM. (1999). Orphanet: an online rare disease and orphan drug data base. Malignant triton tumor. ORPHA:252212

Malignant triton tumor (MTT) is a rare aggressive subtype of malignant peripheral nerve sheath tumor (MPNST; see this term) characterized histopathologically by focal rhabdomyoblastic differentiation. 